\title{
Extending the Network Lifetime by Distributed Selection of Cluster Head in Homogeneous Wireless Sensor Networks
}

\author{
Bahram Moradi Nezhad $^{1}$, Mojtaba Alizadeh $^{2}$, Touraj Khodadadi ${ }^{3}$ \\ ${ }^{1}$ Computer Engineering Department, Lorestan University, Khorramabad, Iran, cert@lu.ac.ir \\ ${ }^{2}$ Computer Engineering Department, Lorestan University, Khorramabad, Iran, alizadeh.mo@lu.ac.ir \\ ${ }^{3}$ Department of Information Technology, School of Science and Engineering, Malaysia University of Science and \\ Technology, Malaysia, touraj@must.edu.my
}

\begin{abstract}
Recently, the integrated circuit manufacturing technologies are developing very fast, which gives the opportunity to the wireless sensor networks to get more popular. As the use of this technology is risen, managing of this network become a challenging research area. Routing protocol has a key role to improve management mechanism in wireless sensor networks. This paper is focused on clustering of wireless sensor networks and tries to resolve the problems on node clustering by optimizing current methods. Hence, a suitable clustering protocol is proposed to increase the network lifetime, data transport throughout and optimize load balancing. To achieve this goal various criteria such as node remaining energy, distance between nodes, density, and auxiliary cluster head are considered in the proposed optimized method. Furthermore, the proposed method is analyzed and evaluated via simulation using NS2 and compared to the related works. The results of the simulation prove the improvement of the proposed method.
\end{abstract}

Key words: Extending Life time, Cluster heads distribution, Load balancing, Wireless sensor networks

\section{INTRODUCTION}

Wireless sensor networks (WSNs) are a new class of telecommunication networks, which help us to collect physical environment statistics without human attendance. WSNs provide us with new insights to improve our life quality via developing new applications. In general, WSN can do the tasks, which had been impossible before. By emergence of new standards, and systems with minimum energy consumption, application of these networks will grow even further [1]-[6]. However, WSNs suffer from some drawbacks and some changes must be made in their management schemes to optimize the current management schemes and resolve the current issues. Among these changes, variations in security, clustering and routing methods can be mentioned [7]-[16]. Nodes' clustering by new techniques is one of the effective solutions to achieve to the goals such as energy consumption management. By application of clustering techniques, energy limitation challenges can be solved significantly in wireless sensor networks. Nodes' grouping in the clusters has been widely accepted in research communities to achieve to scalability and in general higher energy efficiency and longer lifetime in WSN environments [17]- [22].

In the hierarchical structure of the networks, each network has a leader called cluster head that is responsible for specific tasks such as data fusion and aggregation. Each cluster also has several ordinary nodes as its members and the main duty of the cluster head is to collect data from the cluster nodes [23]-[25]. After data collection from its sub-nodes, the cluster head sends the data to the main center for analysis and processing; the data can also be guided to the main center through cluster head in a stepwise manner. Node clustering can drastically reduce the communicative costs of the nodes and several clustering protocols have been proposed for WSNs, which shows proper efficiency in their working area [26]- [29].

Distributive Energy Efficient Adaptive Clustering (DEEAC) algorithm is a clustering method, which classifies the sensor nodes into the groups. In this way, sensor nodes deliver the data to their cluster head and the cluster head will deliver the gathered data to its reference station. This method will save the nodes' energy and therefore increase the network lifetime. This protocol is inspired from LEACH protocol, which is a probability-based clustering including spatial and temporal variables about the data reports from the entire network. In LEACH protocol, a cluster head will be selected based on probability; therefore, the energy consumption of the networks will not be uniform throughout the network; hence it will increase energy consumption and decrease the network lifetime. In contrast, in this probability-based method, temperature and heat of the region will be considered to select cluster head to use the similar application of the nodes and energy consumption distribution. In addition to LEACH parameters, parameters such as candidate cluster head node energy dissipation is used to determine a value for each node at different temperatures. Then, based on this value, Hotness of a cluster head will be selected for optimal energy consumption. This can be achieved by creation of a number of threshold adaption (T) in LEACH algorithm [30]. 
Fuzzy Based Energy Efficient Multiple Cluster Head Selection Routing Protocol (FEMCHRP) algorithm is a clustering method, which carries out both network routing and clustering. Sending process in this algorithm involves data collection by use of fuzzy logic from sensor nodes; then a group of nodes will be considered as a cluster one of which will be selected as the cluster head based on shortest distance and maximum energy level. Finally, the cluster head node will transfer the collected data to the leader cluster head, where they will be sent to base station (BS). Finally, Dijkstra algorithm selects the best route, which consumes the least amount of energy. The main goal of this algorithm is to increase the network lifetime and balance the energy consumption in the network. In case of routing methods, numerous algorithms have been propose based on single-step routing, multiple-step routing, fuzzy logic, and position-based routing. However, these methods are dependent based on leading cluster head selection that directly sends the collected data to the base station. This algorithm mainly follows an efficient data transfer process based on cluster heads and leading cluster heads for WSN [31].

Hierarchical Energy-Balancing Multipath (HEBM) algorithm is a distributed clustering method, which carries out the routing in addition to clustering. In this algorithm, the sink position is predetermined and fixed. This algorithm selects the cluster head node periodically and in a distributed manner based on several parameters such as sensing node weight, its energy, and distance of cluster head with its neighbors, node distance with the sink. The goal of this algorithm includes energy efficiency, increase of lifetime, distributed cluster head selection, hierarchical clustering and load balance [32]-[33].

Based on the literature, node clustering for better routing mechanism need to be optimized. Henceforth, Extending the Network Lifetime by Distributed Selection of Cluster Head Protocol (ENLDSCHP) is proposed in this paper. Different phases of the proposed method are discussed in the following section. This method covers the related work drawbacks.

\section{PROPOSED METHOD}

ENLDSCHP algorithm is a distributed clustering method for homogeneous wireless sensor networks which selects the cluster head node periodically and in a distributed manner. The main goal of the proposed method is to increase the network lifetime, load balance, efficiency and optimal energy consumption, increase the data transport and minimize and terminate the fixed-number node clustering, provide distributed clusters and compact groups based on several parameters including maximum sensor node energy level, minimum distance of sensor node, maximum sensor node density, shortest distance of cluster head node with the sink, maximum energy level of the sink, selection of auxiliary cluster head by consideration of 5 above parameters.

In ENLDSCHP protocol, during operational phase of the network, a series of calculations will be carried out for selecting the best cluster head and auxiliary cluster head and the best sink for data transport from member nodes. This protocol has 5 steps:

1- First each of these nodes sends a message (package) including spatial position of the node to its neighbors.

2-Each node activates a counter for obtaining its neighbor numbers after receiving the message (package) of spatial position.

3-Each node that has its own and neighbor position, calculates distance sum via Equation 1:

$d i j=\sqrt{(\mathrm{xi}-\mathrm{xj})^{2}+(\mathrm{yi}-\mathrm{yj})^{2}}[26](1)$

4-By equations 2, 3 and 4, merit value can be obtained and based on the obtained results, a merit value will be determined for becoming cluster head. Each node's merit implies that the node has maximum energy and minimum distance with the neighbors and maximum density and minimum distance with sink and maximum sink energy.

The best node will be selected by these three formulas. The best node has maximum energy and density and minimum distance with the neighbors and sink.

$$
\mathrm{f}=\frac{\text { E current }}{\text { dis sum }}[26]
$$

where E current is the current energy level of the sensor node and dis sum represents the sum of sensor node distance with its neighbors. If the merit value of the each of neighboring nodes is less than the selected node's merit value, its probability to be chosen as the cluster head will increase and vice versa, if the merit value of each of the neighboring nodes is more than the selected node, the probability of its selection as the cluster head will be reduced.

$\mathrm{f} 1=\left(\frac{\text { E current }}{\mathrm{E} \max }\right)+\left(1-\frac{\text { dis sum }}{\mathrm{N} * \text { dis max }}\right)[26](2)$

$\mathrm{E}_{\max }$ shows the maximum node energy and dis sum represents the sum of distances to the neighbors. $\mathrm{N}$ denotes the number of neighbors and dis max is the maximum distance to the neighbors. Equation 4 is also used for determination of the best cluster head node which has the shortest distance to the sink. The sink also has the maximum energy level.

$$
\mathrm{f} 2=\left(1-\frac{\text { dis Sink }}{\text { dis furthest Sink }}\right)+\left(\frac{\text { E current Sink }}{\text { E max Sink }}\right)[26]
$$

here, dis sink shows the sink distance to the node, dis furthest Sink represents the maximum sink distance to the node and E Current Sink and E Max Sink are current and maximum energy of the sink, respectively.

5- After consideration of the main cluster head, an auxiliary cluster head will be considered for the main one. The first node with the closest merit value to the cluster head, will be selected as the auxiliary cluster head. 
In the proposed algorithm, by application of the above equations for each node, a merit value will be determined based on a series of parameters mentioned above. Among these nodes, the node with maximum energy level and minimum distance to the neighbors and the sink (the sink also has the maximum energy level.

\section{SIMULATION AND ANALYSIS}

For simulation of this clustering algorithm, a network was designed in ns 2 software. Then 100 nodes and a series of initial parameters were defined for the simulation. The simulation environment is shown in Figure 1 and Figure 2. The simulation parameters and specification are described in Table 1 and Table 2.

Table 1: Parameters of simulation

\begin{tabular}{|l|c|}
\hline \multicolumn{1}{|c|}{ Parameter } & Value \\
\hline Area & $\mathbf{2 0 0} \mathbf{m} \times \mathbf{~ 2 0 0 m}$ \\
\hline Data packet size & $4000 \mathrm{bits}$ \\
\hline Control packet size & $512 \mathrm{bits}$ \\
\hline Number of sensor nodes & $100 / 200 / \ldots . / 800$ \\
\hline Initial energy & $2 \mathrm{~J}$ \\
\hline Base station location & $(50.50)$ \\
\hline Distance $d_{\underline{0}}$ & $87 \mathrm{~m}$ \\
\hline$E_{\text {elec }}$ & $50 \mathrm{nj} / \mathrm{bit}$ \\
\hline
\end{tabular}

Table 2: Simulation Specifications

\begin{tabular}{|l|c|}
\hline \multicolumn{1}{|c|}{ Specification } & Value \\
\hline Channel Type & Wireless \\
\hline Radio Propagation Model & Double Mode \\
\hline Network Connector Type & Wireless Phy \\
\hline MAC Type & Mac/802_11 \\
\hline Queue Connector Type & $\begin{array}{c}\text { Queue/Drop Tail/Pri } \\
\text { Queue }\end{array}$ \\
\hline Link Layer Type & LL \\
\hline Coverage Type & Antenna/Omni,Antenna \\
\hline Maximum Packet & 50 \\
\hline Node Number & 100 \\
\hline Routing Protocol Type & AODV \\
\hline Simulation Time Span & $700 \mathrm{~s}$ \\
\hline
\end{tabular}

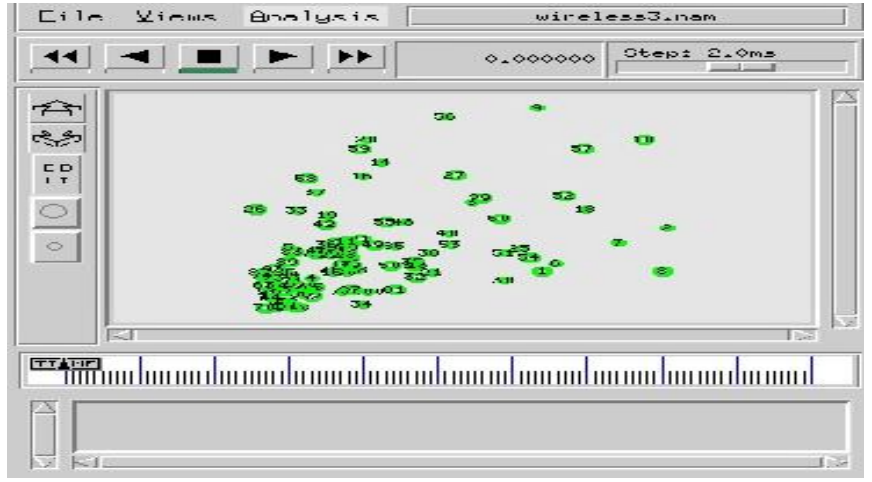

Figure 1: Simulation Environment

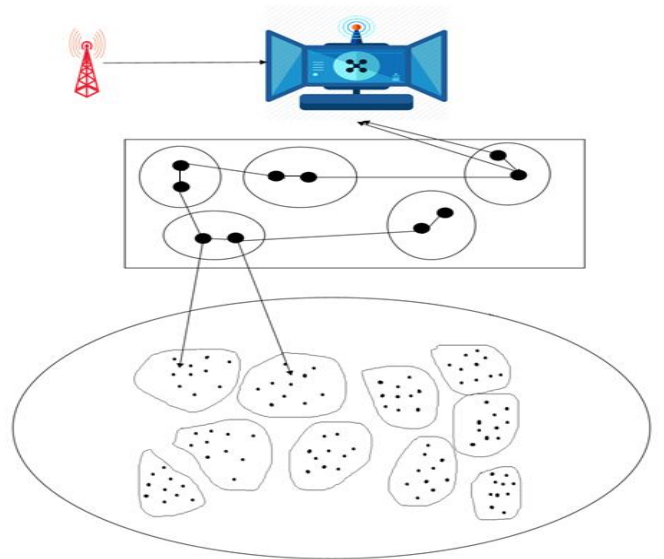

Figure 2: ENLDSCHP Network transmission

\subsection{Results Analysis}

This section analyzes the results of the proposed method and compares to the related works. Each analysis is described in a separate figure as follows.

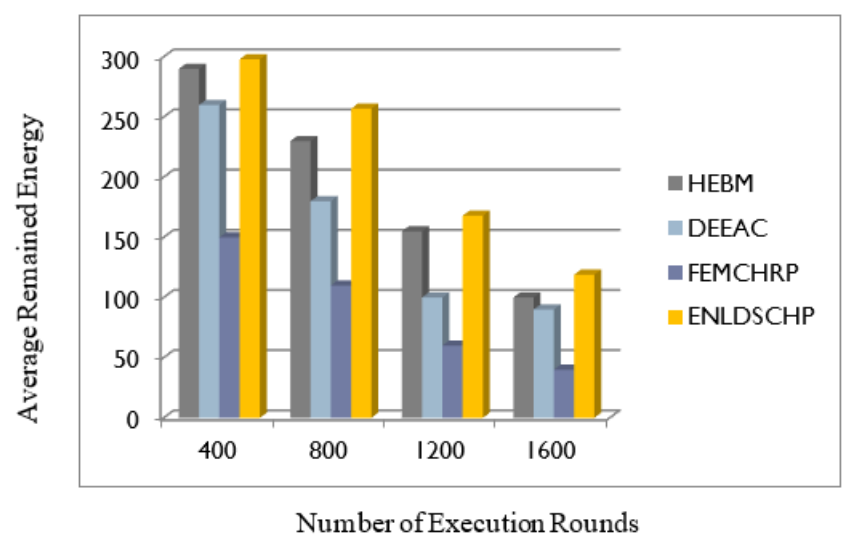

Figure 3: Average remained energy

The remaining energy of each sensor for the proposed method compared to the related works in Figure 3. This figure shows that the level of the remaining energy of the proposed method is higher than the other method. Furthermore, energy consumption as an important criterion is evaluated in Figure 4. 
The proposed method consumes the lowest amount of energy compare to other methods such as HEBM, FEMCHRP and DEEAC algorithms.

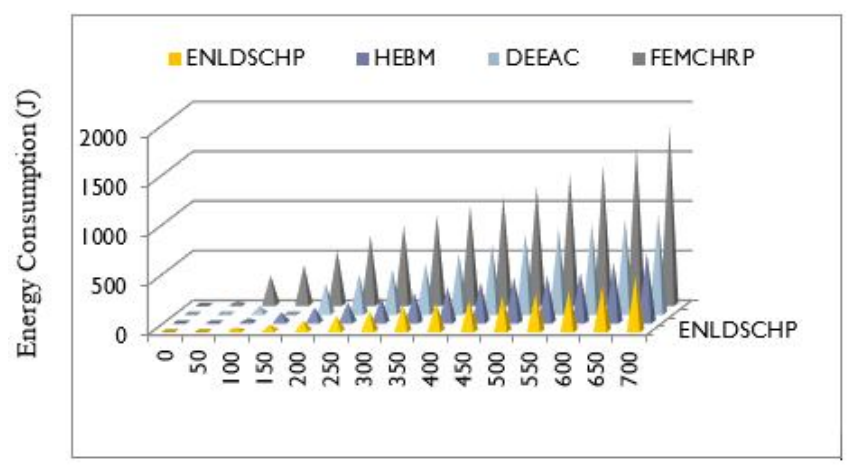

Figure 4: Energy consumption comparison

Resource limitations of wireless sensors cause reduction of sensors lifetime; hence, routing protocols should consume less energy to have bigger lifetime. According to Figure 5, Figure 6, and Figure 7 compare lifetime of sensors in the proposed method to the related works, which confirms the proposed method offers better lifetime span compared to other methods.

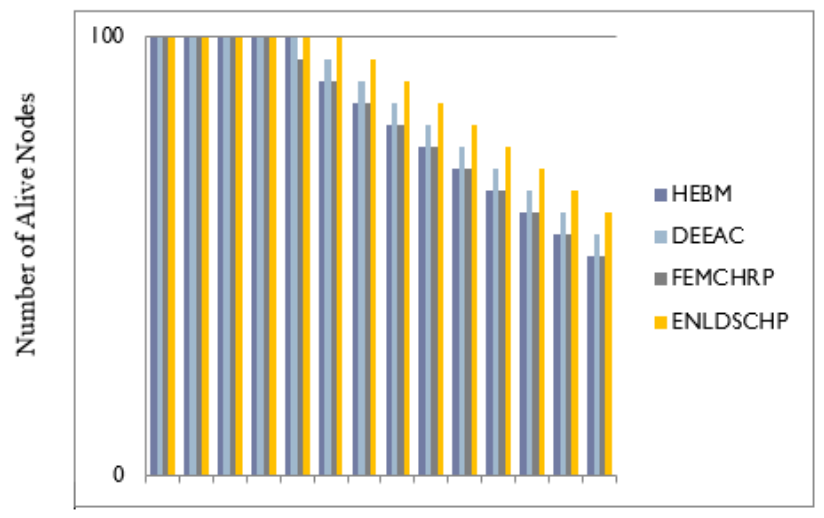

Time (S)

Figure 5:Lifetime span (number of alive nodes)

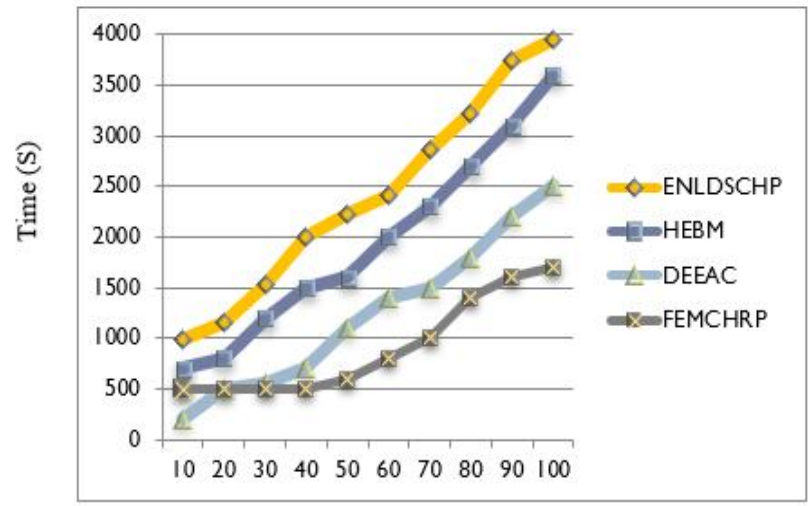

Dead Nodes Percentage

Figure 6:Dead nodes percentage

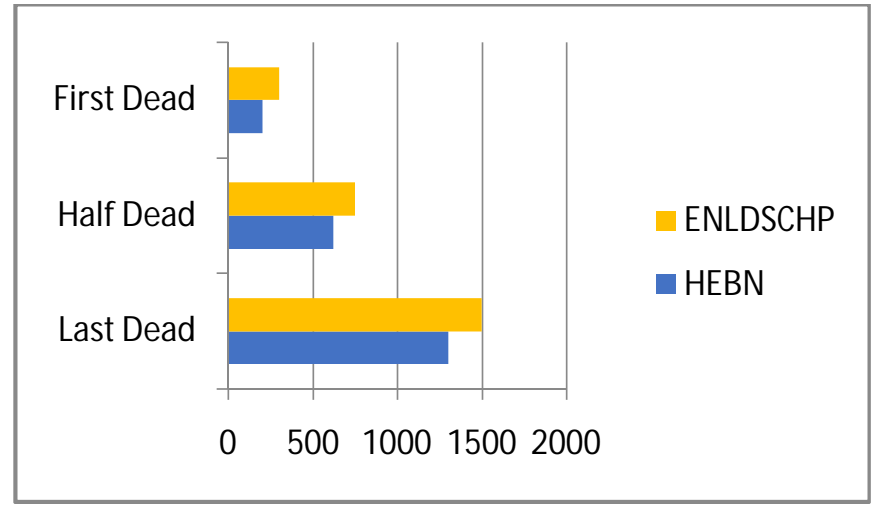

Figure 7:The competition between dead nodes in first, middle and last time

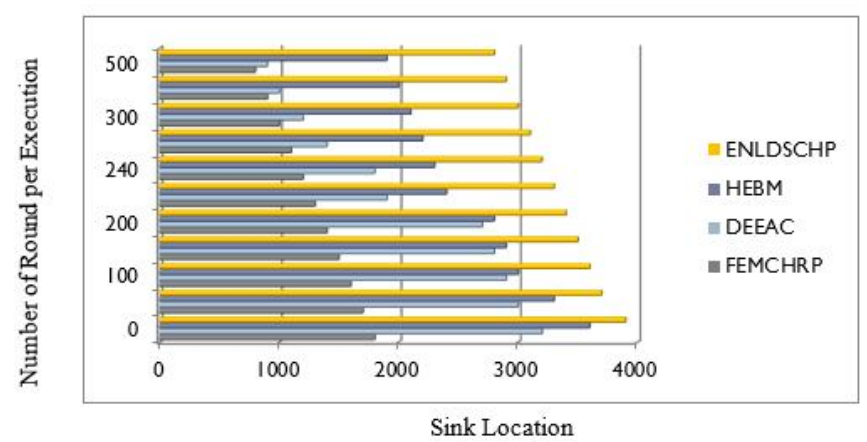

Figure 8: The location of the sink vs. the network lifetime

Based on the results of Figure 8, the proposed method offers better network lifetime in various locations of the sink. According to this figure, sink location affects the network lifetime directly.

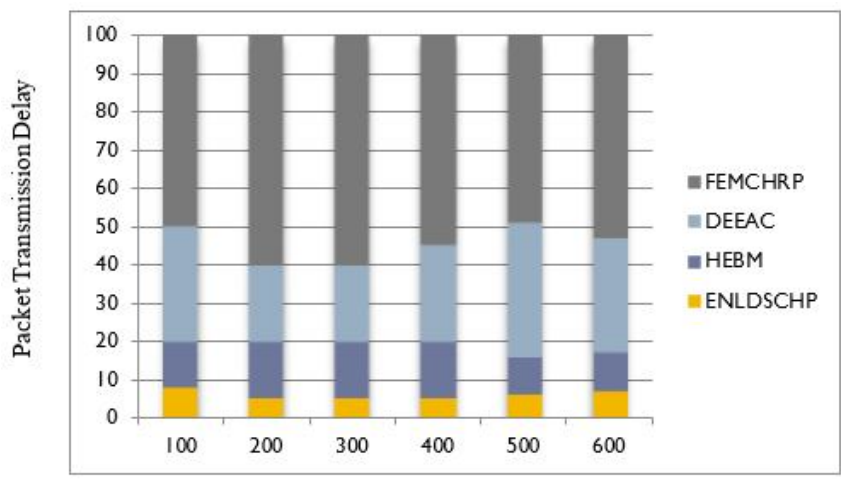

Number of Nodes

Figure 9: The delay of packet per more nodes measure

Figure 9 shows that the packet delay of the proposed method is less than other related works for different number of nodes. Based on this figure, the proposed method shows better performance in case of different number of nodes.

After simulation, it was found that ENLDSCHP clustering protocol can play a crucial role in energy consumption of WSNs. Regarding the output curves and tables of the simulated algorithms mentioned above, this protocol managed 
to increase the network lifetime, reduce the energy consumption, balance the load, reduce the network excess load, increase the network transport and some other parameters to improve the network management. In this way, this protocol succeeded to manage one of the important challenges of WSNs (energy management in clustering operation) more optimally than FEMCHRP, DEEAC, HEBM protocols. The comparative analysis the proposed method and other three related works including DEEAC, FEMCHRP, and HEBM, is provided in Table 3.

Table 3: Comparative Analysis of three clustering protocols (DEEAC, FEMCHRP, HEBM and ENLDSCHP)

\begin{tabular}{|l|l|c|c|c|}
\hline \multicolumn{1}{|c|}{ Parameter } & DEEAC & FEMCHRP & HEBM & ENLDSCHP \\
\hline Extending Life time & Medium & Low & Medium & High \\
\hline Load balancing & Medium & Low & Good & High \\
\hline Energy efficiency & Medium & Low & Medium & High \\
\hline Delivery delay & Medium & High & Low & Small \\
\hline Scalability & Medium & Low & Medium & High \\
\hline Packets sent to $(B S)$ & Low & Low & Medium & High \\
\hline
\end{tabular}

\section{CONCLUSION}

This paper focuses on clustering of wireless sensor networks and tries to resolve the problems on node clustering by new techniques. In this regard, a clustering protocol was proposed to achieve objectives such as increasing the network lifetime, load balancing and increase of data transport through periodic and distributed selection of cluster heads and sinks according to nodes' remaining energy, their distance, density and auxiliary cluster head. The simulation results prove that the proposed method optimized energy consumption of WSN compared to the current methods. Furthermore, other criteria such as network lifetime, load balancing, and network overhead are optimized compare to HEBM, DEEAC, and FEMCHRP algorithms.

\section{REFERENCES}

1. N. Nokhanji, Z. M. Hanapi, S. Subramaniam, and M. A. Mohamed."An energy aware distributed clustering algorithm using fuzzy logic for wireless sensor networks with non-uniform node distribution,"Wireless Personal Communications, vol. 84, no. 1, pp. 395-419, 2015. https://doi.org/10.1007/s11277-015-2614-9

2. N. L. Sowjanya, \& R. Anitha. An Efficient VLAN Implementation to decrease Traffic Load in a Network.International Journal of Advanced Trends in Computer Science and Engineering, 9(2), 2020. https://doi.org/10.30534/ijatcse/2020/189922020

3. S. Sairohith, B. Naga Kalki Venkatesh, Dr. JKR Sastry, J. Rajasekhar.Inter-Networking Heterogeneous Embedded Networks through Universal Bus.International Journal of Advanced Trends in Computer Science and Engineering. 9(2), 2020. https://doi.org/10.30534/ijatcse/2020/80922020
4. A. A. Bara'a, and E. A. Khalil."A new evolutionary based routing protocol for clustered heterogeneous wireless sensor networks,"Applied Soft Computing, vol. 12, no. 7, pp. 1950-1957, 2012. https://doi.org/10.1016/j.asoc.2011.04.007

5. M. O. Oladimeji, M. Turkey, and S. Dudley."A heuristic crossover enhanced evolutionary algorithm for clustering wireless sensor network."In European Conference on the Applications of Evolutionary Computation, pp. 251-266.

6. E. Amiri, H. Keshavarz, M. Alizadeh, M. Zamani, and T. Khodadadi."Energy Efficient Routing in Wireless Sensor Networks Based on Fuzzy Ant Colony Optimization,'International Journal of Distributed Sensor Networks, vol. 2014, pp. 1-17, 2014. https://doi.org/10.1155/2014/768936

7. Y. Huang, J.S Chen, L.MWang. Performance of Transmission Delay and Energy Consumption of Adaptive Listening Mechanism for Wireless Sensor Networks. International Journal of Advanced Trends in Computer Science and Engineering. 8(6), 2019. https://doi.org/10.30534/ijatcse/2019/88862019

8. M. Eshaftri, A. Y. Al-Dubai, I. Romdhani, and M. B. Yassien. "A new energy efficient cluster based protocol for wireless sensor networks." pp. 1209-1214.

9. C. Wei, J. Yang, Y. Gao, and Z. Zhang."Cluster-based routing protocols in wireless sensor networks: a survey."In Proceedings of 2011 International Conference on Computer Science and Network Technology, pp. 1659-1663.

10. J. N. Al-Karaki, and A. E. Kamal."Routing techniques in wireless sensor networks: a survey,'IEEE wireless communications, vol. 11, no. 6, pp. 6-28, 2004.

11. K. Akkaya, and M. Younis. "A survey on routing protocols for wireless sensor networks,'Ad hoc networks, vol. 3, no. 3, pp. 325-349, 2005.

12. E. Gaura, L. Girod, J. Brusey, M. Allen, and G. Challen.Wireless sensor networks: Deployments and design frameworks,Springer Science \& Business Media, 2010. https://doi.org/10.1007/978-1-4419-5834-1

13. R. Cerulli, R. De Donato, and A. Raiconi.'Exact and heuristic methods to maximize network lifetime in wireless sensor networks with adjustable sensing ranges"European Journal of Operational Research, vol. 220, no. 1, pp. 58-66, 2012.

14. R. Han, W. Yang, and K. You."MB-OFDM-UWB based wireless multimedia sensor networks for underground coalmine: a survey,'Sensors, vol. 16, no. 12, pp. 2158, 2016.

15. J. Huang, Q. Duan, C.-C. Xing, and H. Wang."Topology control for building a large-scale and energy-efficient internet of things,'IEEE Wireless Communications, vol. 24, no. 1, pp. 67-73, 2017. https://doi.org/10.1109/MWC.2017.1600193WC

16. M. M. Afsar, and M.-H. Tayarani-N."Clustering in sensor networks: A literature survey,"Journal of Network and Computer Applications, vol. 46, pp. 198-226, 2014. 
17. L. Kong, Q. Xiang, X. Liu, X.-Y. Liu, X. Gao, G. Chen, and M.-Y. Wu.'ICP: Instantaneous clustering protocol for wireless sensor networks,"Computer Networks, vol. 101, pp. 144-157, 2016.

18. P. Zhang, G. Xiao, and H.-P. Tan."Clustering algorithms for maximizing the lifetime of wireless sensor networks with energy-harvesting sensors,"Computer Networks, vol. 57, no. 14, pp. 2689-2704, 2013. https://doi.org/10.1016/j.comnet.2013.06.003

19. P. J. Kaur."A survey of clustering techniques and algorithms."In 2015 2nd international conference on computing for sustainable global development (INDIACom), pp. 304-307.

20. F. Kiani, E. Amiri, M. Zamani, T. Khodadadi, and A. Abdul Manaf. Efficient intelligent energy routing protocol in wireless sensor networks.International Journal of Distributed Sensor Networks, 11(3), $618072,2015$.

21. S. Naeimi, H. Ghafghazi, C.-O. Chow, and H. Ishii."'A survey on the taxonomy of cluster-based routing protocols for homogeneous wireless sensor networks,'Sensors, vol. 12, no. 6, pp. 7350-7409, 2012.

22. V. Pal, G. Singh, and R. Yadav.'Balanced cluster size solution to extend lifetime of wireless sensor networks,'IEEE Internet of Things Journal, vol. 2, no. 5, pp. 399-401, 2015.

https://doi.org/10.1109/JIOT.2015.2408115

23. M. Alizadeh, M. H. Tadayon, K. Sakurai, S. Baharun, H. Anada, M. Zamani, A. Zeki, S. A. Chaudhry, and M. K. Khan."Comments and improvements of "HOTA: Handover optimized ticket-based authentication in network-based mobility management", Information Sciences, pp. 112-116.

24. M. Alizadeh, S. Abolfazli, M. Zamani, S. Baharun, and K. Sakurai."Authentication in Mobile Cloud Computing: A Survey,"Journal of Network and Computer Applications, vol. 61, pp. 59-80, 2016.

25. B. Abarghouei, A. Farokhtala, and M. Alizadeh.'DNACK: False Data Detection Based on Negative Acknowledgment and Digital Signature on Mobile Ad-hoc Network,"Wireless Personal Communications, vol. 83, no. 1, pp. 1-15, 2015/05/29, 2015. https://doi.org/10.1007/s11277-015-2327-0

26. C. Guy."Wireless sensor networks:a survey",Computer networks,p. 63571I.

27. I. F. Akyildiz, and M. C. Vuran.Wireless sensor networks, John Wiley \& Sons, 2010.

28. J. Han, J. Pei, and M. Kamber, Data mining: concepts and techniques, Elsevier, 2011.

29. A. Chakraborty, S. K. Mitra, and M. K. Naskar.“Energy efficient routing in wireless sensor networks: A genetic approach,”arXiv preprint arXiv, 1105.2090, 2011.

30. I. Eyal, I. Keidar, and R. Rom.'Distributed data clustering in sensor networks,'Distributed computing, vol. 24, no. 5, pp. 207-222, 2011. https://doi.org/10.1007/s00446-011-0143-7

31. S. Rana, A. N. Bahar, N. Islam, and J. Islam.'Fuzzy based Energy efficient multiple cluster head selection routing protocol for wireless sensor networks,"International Journal of Computer Network and Information Security, vol. 7, no. 4, pp. 54, 2015. https://doi.org/10.5815/ijcnis.2015.04.07

32. C. Gherbi, Z. Aliouat, and M. Benmohammed."An adaptive clustering approach to dynamic load balancing and energy efficiency in wireless sensor networks,"Energy, vol. 114, pp. 647-662, 2016. https://doi.org/10.1016/j.energy.2016.08.012

33. M. Eslami, O. Karimi, O, and T. Khodadadi. A survey on wireless mesh networks: Architecture, specifications and challenges. In 2014 IEEE 5th Control and System Graduate Research Colloquium,(219-222), IEEE, 2014. https://doi.org/10.1109/ICSGRC.2014.6908725 